\title{
Multifractal Scaling, Geometrical Diversity, and Hierarchical Structure in the Cool Interstellar Medium
}

\author{
David Chappell and John Scalo \\ Astronomy Dept., University of Texas, Austin, TX 78712
}

\begin{abstract}
Multifractal scaling (MFS) refers to structures that can be described as a collection of interwoven fractal subsets which exhibit power-law spatial scaling behavior with a range of scaling exponents (concentration, or singularity, strengths) and dimensions. The existence of MFS implies an underlying multiplicative (or hierarchical, or cascade) process. Panoramic column density images of several nearby star-forming cloud complexes, constructed from IRAS data, are shown to exhibit such multifractal scaling, which we interpret as indirect but quantitative evidence for nested hierarchical structure. The relation between the dimensions of the subsets and their concentration strengths (the "multifractal spectrum") appears to satisfactorily order the observed regions in terms of the mixture of geometries present, from strong point-like concentrations, to line-like filaments or fronts, to space-filling diffuse structures. This multifractal spectrum is a global property of the regions studied, and does not rely on any operational definition of "clouds." The range of forms of the multifractal spectrum among the regions studied implies that the column density structures do not form a universality class, in contrast to indications for velocity and passive scalar fields in incompressible turbulence, providing another indication that the physics of highly compressible interstellar gas dynamics differs fundamentally from incompressible turbulence. There is no correlation between the geometrical properties of the regions studied and their level of internal star formation activity, a result that is also apparent from visual inspection. We discuss the viability of the multifractal spectrum as a measure of the structural "complexity" of the regions studied, and emphasize the problematic dependence of all structural descriptors on the subjective pre-selection of the region to be described. A comparison of IRAS $100 \mu \mathrm{m}$ column density (not intensity) images with ${ }^{13} \mathrm{CO}$, visual extinction, and $\mathrm{C}^{18} \mathrm{O}$ data suggests that structural details are captured by IRAS up to at least 30 magnitudes of visual extinction, except in the vicinity of embedded stars, and that lower-column density connective structure not seen by other methods is revealed.
\end{abstract}

Subject headings: galaxies: ISM — ISM: clouds, structure - methods: data analysis - techniques: image processing - turbulence

\section{Introduction}

Multifractal scaling (MFS) is a property that refers to measures or fields for which the local intensity (e.g. density, column density, radial velocity, temperature), integrated out to distance $r$ from a given position, scales as a power law $r^{\alpha}$, with the scaling exponent $\alpha$ varying from position to position. If the collection of positions with scaling exponents in $(\alpha, \alpha+\mathrm{d} \alpha)$ can be described as a fractal with (Hausdorff) dimension $f(\alpha)$, for a range of $\alpha$, then the structure is multifractal, and the function $f(\alpha)$ is called the multifractal spectrum (MFS; we use MFS for both "multifractal scaling" and "multifractal spectrum" when no ambiguity can arise). This definition is not unique in the literature, but is sufficient for our purposes (see Feder 1988, McCawley 1990, Muzzio et al. 1992). The exponent $\alpha$ is called the "singularity strength" in the multifractal literature, since 
the power law in principle gives a density singularity as $r$ approaches zero; here we prefer to use the more descriptive term "concentration strength" for this exponent. Similarly, the multifractal spectrum is often referred to as the "spectrum of singularities" in the physics literature, but we avoid this terminology here; however, since we do use the potentially misleading term "multifractal spectrum", it is important to remember that " $f$ " is a dimension of a subset, not a filling factor.

The MFS basically contains information on how the geometry, as measured by the dimension, depends on the concentration strength $\alpha$; loosely speaking, the $f(\alpha)$ curve shows the manner in which regions of different concentration strength fill space. For example, for subregions with a given $\alpha$, the dimension will be different for point-like, line (or filament)-like, or space-filling structures. So the MFS indicates the distribution of geometries present in a complex structure. For a homogeneous fractal the $f(\alpha)$ curve collapses to a point; otherwise the measure (intensity image) is multifractal, since regions of different concentration strength have different dimensions. A common $f(\alpha)$ curve between physical processes and/or mathematical models can be interpreted as evidence that they belong to the same "universality class", 1 at least with respect to the $f(\alpha)$ function. However, it is well-known that the $f(\alpha)$ descriptor (and all other structural descriptors of which we are aware) are degenerate with respect to the underlying physics, in the sense that systems with very different underlying dynamics can exhibit identical $f(\alpha)$ curves within the measurement uncertainties; see Bulajich and Perez-Pascual (1991), also Chhabra, Jensen, and Sreenivasan (1989). We give a simple example in Appendix B.

Multifractal scaling usually occurs in systems that arise from multiplicative processes, or "cascades," in which the intensity (e.g. density, etc.) at a given position is due to successive multiplications along a hierarchical tree (see below), so the existence of MFS (and its properties) can be used to infer hierarchical (nested) structure, and to constrain the types of physical processes that give rise to such a structure. The present work attempts to test for MFS in column density maps of nearby cloud complexes and to find whether the resulting $f(\alpha)$ function can be used to describe the mixture of geometries present in these structures.

Densely sampled extinction, molecular line, H I line, and IRAS maps of nearby cloud complexes have shown that the gas and dust are organized into complicated structures, with irregularities present over a wide range of scales, from roughly 0.02 parsecs (resolution limit for the nearest cloud complexes) to at least a few hundred parsecs (see reviews in Scalo 1985, 1990, Wilson and Walmsley 1989, Falgarone and Phillips 1991, Stutzki et al. 1991, Genzel 1991, Falgarone and Perault 1992, Elmegreen 1992, and references therein). The geometry and scaling of this structure may provide a signature of the physical processes that are involved in star formation, and may be related to important quantities such as the star formation efficiency and the initial mass function, as suggested by Henriksen (1991) and Larson (1992). Elmegreen and Falgarone (1996) and Elmegreen (1997) have proposed that the fractal scaling properties of the interstellar medium (ISM) are consistent with many of its observed features, including the mass-size scaling, the cloud mass spectrum, and the existence and properties of the intercloud medium. Scaling behavior is the basis for physical interpretation in a huge number of applications within physics, with phase transitions and turbulence being most prominent.

Since there is no unique way to characterize structure, however, there are many ways to define structural scaling. One such method is the perimeter-area relation (see Feder 1988, chap. 12). If contours of equal intensity (or column density, or temperature, etc.) exhibit a power law perimeter-area relation with a non-integer exponent over a range of scales, this exponent may be interpreted in terms of a fractal dimension which characterizes the manner in which these curves fill space. A number of studies have demonstrated such behavior in local interstellar cloud

${ }^{1}$ For example, in turbulence the MFS is believed to be "universal" at the onset of chaos, because it agrees with the MFS of the 1-dimensional circle map. 
structures structures:2 using extinction maps (Beech 1987, Hetem and Lépine 1993), H I maps (Wakker 1990), $100 \mu \mathrm{m}$ dust intensity or column density maps (Bazell and Desert 1988, Scalo 1990, Dickman, Horvath, and Margulis 1990, Vogelaar, Wakker, and Schwarz 1991) and CO emission maps (Falgarone, Phillips, and Walker 1991); see also Vogelaar and Wakker (1994). Surprisingly, these studies find a perimeter-area dimension of about 1.3-1.5 for most clouds studied, similar to the value found for terrestrial cloud and rain areas (Lovejoy 1982) and slices of laboratory turbulence (see Sreenivasan 1991 for a review). Even the HI gas in the M81 group of galaxies appears to exhibit a very similar perimeter-area dimension (Westpfahl et al 1999). This result suggests a sort of universality for cloud fractal geometry which is independent of the presence of young stars or the importance of self-gravity, and may be related to turbulence (by which we mean phenomena associated with nonlinear advection), as emphasized by Scalo (1990) and Falgarone et al. (1991), or some other process, since the dimension of a fractal is extremely degenerate with respect to underlying processes and morphological appearance (i.e. many very different processes and forms can give rise to the same dimension).

In the present work we are concerned with a different type of scaling behavior, and it is important to appreciate the difference. If the intensity (or column density, or radial velocity, or any measured quantity) image is represented as a surface whose local height is the intensity ("mountain range," or "topographic," representation), then the dimension obtained from the perimeter-area relation characterizes the irregularity of contours formed by horizontal cuts through the "mountain range." However, this dimension does not uniquely or completely characterize the scaling of the intensity surface itself. Adding a constant or multiplying by any function of the image intensity will not affect the power-law slope of the perimeter-area relation, although, for example, the "spikiness" of the mountain range may be greatly affected. Furthermore, it is commonly stated that if the perimeter-area relation is a power law with exponent $d$, then the clouds have a fractal dimension $D=2 d$. However, this gives the incorrect impression that if this scaling holds then the cloud complex is a statistically self-similar fractal, when in fact perimeter-area scaling only demonstrates that the "mountain range" is a self-affine fractal. [3 The structure of the intensity surface of such self-affine fractals may possess a continuous range of scaling exponents or dimensions.

In the multifractal approach used here, the column density "surface" is not sliced at different intensities. Instead the present technique is equivalent to partitioning the image into different subsets according to the value of the local concentration strength $\alpha$, defined by the exponent of a local power law scaling of the intensity integrated out to distance $r$ from a position, if such a power-law scaling exists. The dimension of this subset is not estimated by treating it as a collection of curves (contour map) and characterizing the irregularity of each curve; the dimension is estimated for the entire subset, treated as a collection of points, and includes all the points, not just those comprising disjoint closed curves. No reduction to "contours" is involved in the process. As will be seen, the dimensions estimated in this way are very different from the perimeter-area dimension, although the relation between the two remains unclear to us. For example, we will show that the column density surface in the Taurus complex (and the other regions we examined) is a multifractal, even though it is known to possess a well-defined perimeter-area relation with a single fractal dimension for all intensities (Scalo 1990, Falgarone et al. 1991). Still, we think that the perimeter-area dimension is a useful and illuminating tool that is complementary to other descriptors. A detailed discussion of the perimeter-area dimension applied to the interstellar medium is given in Dickman et al. (1990), to which the reader is referred for more discussion.

\footnotetext{
${ }^{2}$ We use the term "cloud" loosely to designate an under-resolved observed region of space that appears to contain a coherent density enhancement, recognizing that under sufficient resolution this apparent entity will likely lose its coherence and break up into complex substructure.

${ }^{3}$ Self-similarity refers to structures for which each sub-structure can be obtained from the whole structure by a linear contraction which reduces the original structure by the same scale factor in all coordinates. Self-affine refers to structures whose substructure can be obtained from the original by a linear transformation in which different coordinates (e.g. column density and spatial scale) are contracted by different factors.
} 
(Note, however, that the latter study was based on IRAS intensity images, not column densities as used in the present MFS investigation.)

It is also important to distinguish the structural scaling examined here from the type of power law correlations which have often been discussed for interstellar clouds, e.g. cloud size or mass spectrum, or correlations between density or velocity dispersion and region size. First, these latter correlations are between properties defined for separate entities, such as "clumps within clouds" or certain operationally-defined types of "clouds" located in different star-forming regions or different locations in the galaxy. In contrast, the scaling examined here is a property of a given region as a whole, not a correlation between attributes of any operational entities ("clouds"); the concept of "cloud" does not enter the scaling examined here. Second, the specific form of the quantities whose scaling is being examined here (see Section III below) is directly related to the geometry of the structure, in the sense that they describe how the structure fills space.

The manner in which the discovery of multifractal scaling might be related to physical processes is indicated by similar investigations in other fields. Fully-developed incompressible turbulence in a variety of experimental situations exhibits an apparently universal multifractal singularity spectrum, whether determined for the spatial distribution of the dissipation field (see Sreenivasan 1991, 1996 and references therein) or for the velocity signal (Muzy, Bacry, and Arneodo 1991). Thus the $f(\alpha)$ spectrum may reveal an invariant signature of the underlying nonlinear fluid dynamical processes in incompressible turbulence (e.g. vorticity tilting and stretching in three dimensions); i.e. $f(\alpha)$ may reflect the essential physical processes independently of the type of flow or boundary conditions. However, the process reflected by the MFS may be much more generic. For example, Meneveau and Sreenivasan (1989), Chhabra and Sreenivasan (1992) and others have argued that turbulence is in the same MFS universality class as certain simple multiplicative processes, providing a guide for theoretical modeling. A comparison with direct numerical simulations was given in Hosokawa and Yamamoto (1990). Here we show that such a universal $f(\alpha)$ function does not exist for the density structures of the highly compressible cool interstellar medium, even though the structures are multifractal.

In an extragalactic context, Martinez et al. (1990) suggested that the three-dimensional distribution of galaxies in the CfA survey possesses multifractal scaling, and showed that the corresponding $f(\alpha)$ spectrum could be used to distinguish the Soneira-Peebles hierarchical model (a homogeneous fractal) from the Voronoi tessellation model (a multifractal), even though the correlation function for these two models are very similar. A study of multifractal scaling in cold dark matter simulations, with an emphasis on finite size effects, has been given by Colombi et al. (1992). A number of more recent discussions of the relation between possible multifractal scaling and large-scale galaxy clustering have appeared; see Sylos Labini and Pietronero (1996) and references given there. Multifractal scaling has been discussed in other astrophysical contexts, such as the attempt to relate the multifractal spectrum to the geometry and underlying physics of the solar photospheric magnetic field by Lawrence, Rumaikin, and Cadavid (1993). Outside of astrophysics, the use of the multifractal spectrum as a diagnostic tool is widespread. Examples include various aggregation and coalescence models (see Nagatani 1992), characterization of stages of cancer development through analysis of normal and malignant tissues (Muller et al. 1991), and analysis of protein folding through profiles of solvent accessibilities of amino acid chains (Balafas and Dewey 1995).

Multifractal scaling is closely associated with multiplicative processes (see sec. II below), such as stretching and folding of fluid elements (e.g. Muzzio et al. 1992), and so its existence is sometimes taken as evidence for hierarchical structure (e.g. Balafas and Dewey 1995). .7 Since the hierarchical nature of interstellar cloud structure is not yet adequately established or characterized

\footnotetext{
${ }^{4}$ In contrast, the existence of other types of power-law scaling does not obviously provide evidence for nested or multiplicative structure. For example, if a collection of disks with a power law distribution of sizes is distributed at random on a plane, the distribution is a homogeneous fractal, even though there is no nesting; i.e. the distribution
} 
quantitatively (e.g. Houlahan and Scalo 1992), a search for multifractal scaling in ISM data is of obvious interest. In particular, Meneveau and Chhabra (1990) and O'Brian and Chhabra (1992) have suggested an empirical test for hierarchical processes based on the MFS, which we apply below.

In addition, the MFS may be useful in characterizing complex interstellar structures in terms of the distribution of geometries, since it describes the dimensionality of the subsets as a function of concentration strength. As far as we know, other global descriptors of structure (e.g. the correlation function) are insensitive to geometry, except perhaps for fairly simple structures, like periodically-spaced density fluctuations along a filament. Descriptors can be found to identify local specific forms (e.g. minimal spanning tree for finding filaments, Bhavsar and Ling 1988; line and plane shape detectors based on moments, Luo, Vishniac, and Martel 1996), but these descriptors do not characterize the global geometry in the manner of the MFS.

\section{Data}

We obtained 60 and $100 \mu \mathrm{m}$ IRAS images of four different regions of low-mass star formation from the Infrared Processing and Analysis Center (IPAC). The emission seen at these wavelengths is the radiation of warm dust grains heated by local stars and the galactic radiation field. IPAC estimated and subtracted the local zodiacal background, and we removed the galactic background using a cosecant model profile. Other background models, such as fitting a plane or polynomial to local minima, yielded very similar results. A detailed discussion of these and other points can be found in Verter and Rickard (1998).

The far-infrared emission depends on both the dust temperature and the column density. These two quantities may be separated given the 60 and 100 micron fluxes if it is assumed that all of the dust along a given line of sight is at the same temperature, the dust is optically thin, and that a power-law dust emissivity holds. In such a model, the infrared flux is given by $F_{\lambda}=B_{\lambda}\left(T_{d u s t}\right)\left(1-e^{-\tau}\right)$ where $\tau$ is the dust optical depth and $B_{\lambda}\left(T_{d u s t}\right)$ is the Planck function. The dust optical depth was assumed to vary with wavelength as $\lambda^{n}$, with $n=-1$ or -2 . This relation may be iteratively solved for the temperature and column density for every point in the image.

This method for estimating dust temperatures and column densities assumes that the dust along a given line of sight is at a constant temperature, which biases the column density estimates toward the column density of the warmer dust. For embedded sources this warm dust will be concentrated very near the source (compared to the line of sight depth), constituting a small fraction of the true column density along the line of sight, so the column density will be underestimated, sometimes by a large factor. As a result, embedded protostars and H II regions may appear as holes in the final images. (These artifactual holes may actually provide a useful method for identifying embedded sources, since they are visually very distinct.) For this reason we mostly chose regions of relatively low mass star formation for this study, since there the stars have minimal effect on the column density images. A more sophisticated technique that provides an estimate of temperature variations along the line of sight has been proposed by Xie et al. (1991).

The resulting maps do, however, strongly resemble column density maps based on extinction and molecular line data away from embedded stars (e.g. Langer et al. 1989 for the B5 cloud, Snell et al. 1989 for Heiles Cloud 2 and B18 in Taurus, Jarrett, Dickman, and Herbst 1990 for $\rho$ Oph; below and Appendix A for various subregions in Taurus). We emphasize that this agreement applies to column density maps, and that the intensity maps can appear significantly different; some previous comparisons of IRAS maps with molecular line (Abergel et al. 1995) or extinction

will appear (statistically) self-similar as one "zooms in" on it, but the structure is not hierarchical with respect to nesting. There are many ways to obtain a power law size distribution that do not involve any multiplicative processes. 
(Arce \& Goodman 1999) images have used 60 and $100 \mu \mathrm{m}$ intensity images. The reliability of the column density maps presented here can best be seen by visual comparison with the recent adaptive grid wavelet transform extinction maps for the same regions given by Cambresy (1999).

Four nearby ( 100-200 pc) cloud complexes containing low- to intermediate-mass young stellar objects were chosen for study: Taurus, R Corona Australis, Chamaeleon-Musca, and Scorpius-Ophiuchus. The last three regions were divided into 2, 4 and 6 subregions on the basis of their differing visual appearance and star formation properties. Table 1 lists the positions, estimated distances, number of resolution elements (assuming a resolution of 3 arcmin), and rough estimates of the projected density of young stellar objects, when available, in each subregion. Molecular line maps of these regions with the NANTEN telescope are summarized in Kawamura et al. (1999) and Yonekura et al. (2000); comparisons with earlier work and additional references can be found there. Extinction maps that cover roughly the same areas as examined here can be found in Cernicharo (1991, Taurus), Rossano (1978a, R Cr A), Gregorio-Hetem et al. (1988, Cham-Musca), Rossano (1978b, Sco-Oph), Arce \& Goodman (1999, Taurus), and especially Cambresy (1999, Taurus, Sco-Oph, Cham-Musca, and R Cr A). The star formation activity varies from no detected activity in Cham III to widely distributed low-mass stars (and probably higher-mass B stars - see Walter and Boyd 1991) in Taurus, to dense and probably bound clusters in the $\rho$ Oph core and in the R Cr A core. A discussion of the young stellar populations in parts of these regions with references to the original papers can be found in the review by Zinnecker et al. (1993) and the study by Chen et al. (1997). The gas in the entire Sco-Oph region has been affected by interaction with massive stars (see DeGeus 1988). Figures 1, 2, and 3 show logarithmically scaled column density maps of the Cham-Musca, RCrA, and Sco-Oph regions. An image of Taurus enhanced by unsharp masking is shown in Appendix A. The original Taurus column density image is given in Scalo (1990) and a contour map and a "landscape" representation of the region are in Houlahan and Scalo (1992).

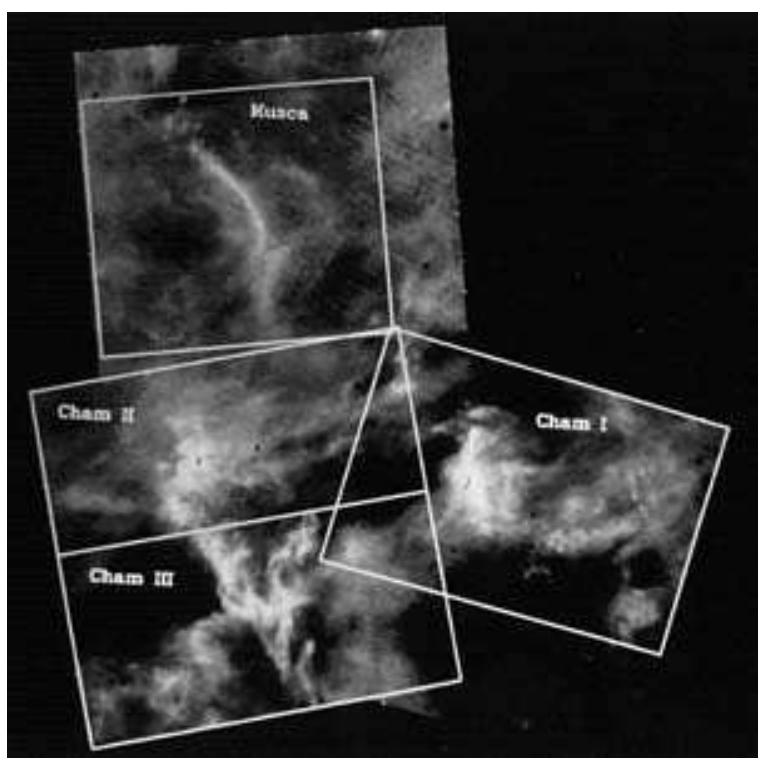

Fig. 1. - Logarithmically scaled image of the column density distribution Chamaeleon-Musca. The small dark holes are embedded protostars which locally heat the dust, yielding an underestimate of the actual column density. The image is a mosaic of three frames, each of which covers $8 \times 8^{\circ}$ on the sky, corresponding to $20 \times 20 \mathrm{pc}$ (assuming a distance of $140 \mathrm{pc}$ ), and contains roughly $6.7 \times 10^{4}$ resolution elements. North is up and west is to the right. 


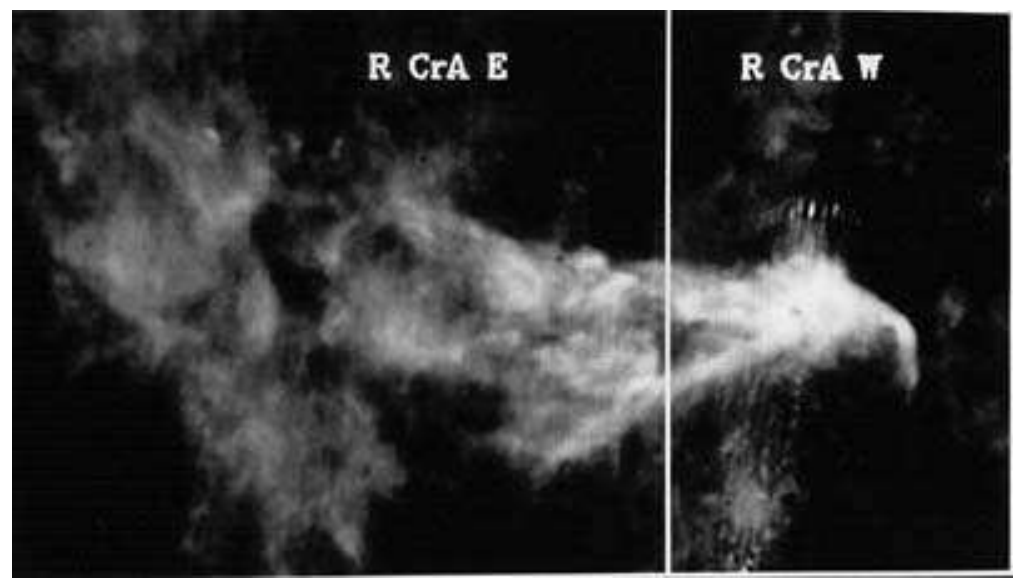

Fig. 2.- Logarithmically scaled image of the column density distribution around R Corona Australis. The image covers $14 \times 8^{\circ}$ on the sky corresponding to $32 \times 18$ pc (assuming a distance of $130 \mathrm{pc}$ ) and contains roughly $4.5 \times 10^{4}$ resolution elements. Residual striping artifacts run vertically through the R CrA W subregion. North is up and west is to the right.

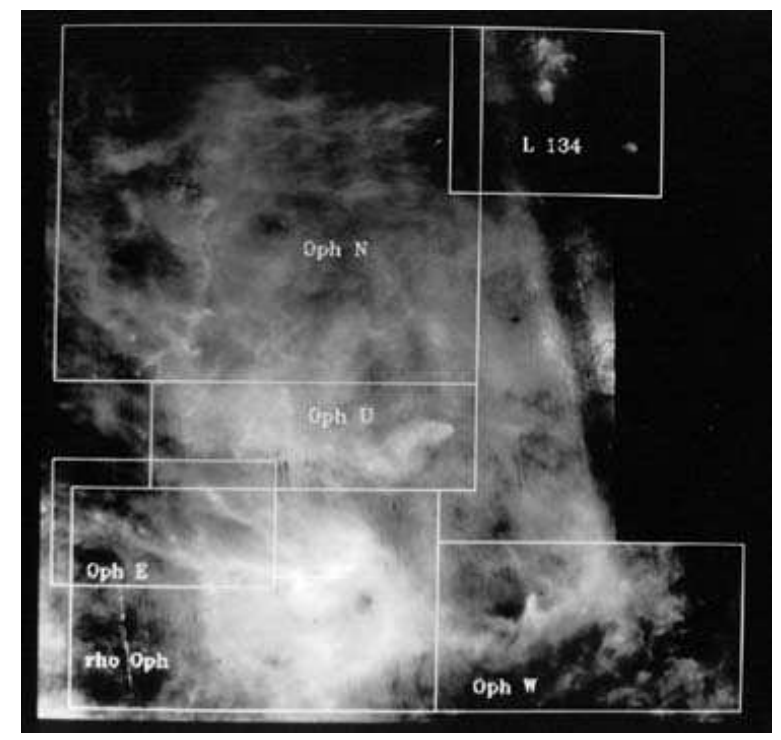

Fig. 3.- Logarithmically scaled image of the column density distribution in the Scorpius-Ophiuchus region. The image covers roughly $28 \times 28^{\circ}$ on the sky corresponding to $61 \times 61 \mathrm{pc}$ (assuming a distance of $125 \mathrm{pc}$ ), and contains $2.5 \times 10^{6}$ resolution elements. The light and dark bands running below and to the right of L134 and east of $\rho$ Oph are residual striping artifacts. North is up and west is to the right.

There is considerable uncertainty concerning the degree to which IRAS $100 \mu \mathrm{m}$ column density $\mathrm{N}_{100}$ represents the true column density. A thorough discussion, with application to cirrus clouds, has been given by Verter and Rickard (1998). Generally the relation between $\mathrm{N}_{100}$ and ${ }^{13} \mathrm{CO}$ column density exhibits a great deal of scatter, yet the resulting spatal images of $\mathrm{N}_{100}$ do closely resemble the structure of ${ }^{13} \mathrm{CO}$ and extinction maps (e.g. Langer et al. 1989 for the B5 
cloud, Snell et al. 1989 for Heiles Cloud 2 and B18 in Taurus, Jarret, Dickman, and Herbst 1990 for $\rho \mathrm{Oph}$ ). Much of the scatter probably arises from a combination of the residual striping, the embedded protostar effect discussed above, and perhaps the background subtraction at the lowest intensity levels. (We point out that the large scatter found in studies like Abergel et al. 1995 is most likely due to the use of IRAS intensities rather than column densities.) However none of the effects seriously compromises the overall spatial structure except in very localized ways, or at the lowest intensity levels. The latter effect forces us to consider only positive moments in the MFS (i.e. the "hole" geometry is not considered - see sec. 4.1 below).

Since the MFS responds to the global structure on all scales, it is the similarity between the spatial structure in $\mathrm{N}_{100}$ and other tracers that is most important. We have compared the images of $\mathrm{N}_{100}$ with available ${ }^{13} \mathrm{CO}$ and extinction maps of five subregions in Taurus, and the results show excellent agreement. An example, the subregion L1529 filamentary substructure in Taurus is shown in Appendix A. The only serious disagreement occurs for the very opaque L1495 subregion. However we find that the $\mathrm{N}_{100}$ image for this subregion does agree much better with the $\mathrm{C}^{18} \mathrm{O}$ image of Duvert, Cernicharo, and Baudry (1986). This suggests that $\mathrm{N}_{100}$ is a better tracer of column density than either ${ }^{13} \mathrm{CO}$ or visual extinction, both of which have severe optical depth effects that set in around $A_{V} \approx 3-5$. The case of L1495 suggests that the upper limit on $A_{V}$ for the validity of $\mathrm{N}_{100}$ may be around $30 \mathrm{mag}$, rather than the 10 mag limit suggested by Jarrett et al. (1989) for the $60 \mu \mathrm{m}$ column density. We also find surprisingly good agreement between the $\rho$ Oph core $\mathrm{N}_{100}$ image derived here and the submillimeter image given by Motte et al. (1998), considering the much better resolution of the submillimeter image, suggesting that the $A_{V}$ limit for $\mathrm{N}_{100}$ (except along lines of sight to embedded protostars) may exceed 50 mag. Thus the IRAS column density upper limit may be similar to that of infrared extinction (e.g. Lada, Alves, and Lada 1999), except that IRAS is locally thwarted by embedded stars, while infrared extinction requires smoothing to reduce noise introduced by structure in the background star positions.

A strong indication of the validity of the IRAS column density images is the excellent visual agreement of our images with the adaptive grid/wavelet decomposition optical extinction maps of several of the regions by Cambresy (1999). Another favorable example is that the "cores" mapped in the $\mathrm{C}^{18} \mathrm{O}$ study of a large part of Ophiuchus by Tachihara, Mizuno, \& Fukui (1999) can be seen to correspond to the highest-column density peaks in the IRAS images of Ophiuchus displayed in Fig.3; the IRAS column density images indicate that the apparently isolated $C^{18} O$ cores studied by Tachihara et al., especially in the regions we refer to as Oph $\mathrm{N}$ and $\mathrm{U}$, are actually connected in a web of elongated or filamentary forms whose column density was too small for the $C^{18} O$ survey.

Overall, these comparisons lead us to be optimistic about the validity of the IRAS column density maps, except near embedded sources or at very low column densities (which we do not use in the subsequent analysis). As remarked above, we found our results to be robust with respect to the adopted power law form of the far-infrared emissivity. Lastly, we found no problems associated with producing seamless mosaics of the IRAS maps, except that it was technically laborious.

We emphasize that a major advantage of the IRAS data is that it can probe a very large range of column densities compared to other methods, roughly from about $2 \times 10^{21} \mathrm{~cm}^{-2}$ (see Weiland eta 1 1986, Verter and Rickard 1998) to $6 \times 10^{25} \mathrm{~cm}^{-2}$ (assuming that the ratio of $A_{V}$ to $\tau_{100}$ is a few thousand).

\section{Calculation of Multifractal Statistics}

The multifractal spectrum is a method of characterizing images and point sets which possess a type of statistical scale invariance called multifractal scaling. The local density profile around a point in the image is characterized by a scaling exponent called the singularity strength (here we use the term "concentration strength"). If $P_{i}(L)$ is the integral of the image intensity (column 
density in the present work) within an aperture of radius $L$ centered at position $i$, then the concentration strength $\alpha$ is defined by $P_{i}(L) \propto L^{\alpha}$. For a two-dimensional homogeneous image $\alpha=2$, while for a sharp spike $\alpha=0$. Thus, the concentration strength measures the local "spikiness" in the image. The function $f(\alpha)$ measures how all the points in the image which have a given concentration strength are distributed and it is the Hausdorff dimension of that point set. The numerical calculation of $f(\alpha)$ has proven to be delicate. Difficulties with procedures used in multifractal analysis have been discussed by Chhabra, Jensen, \& Sreenivasan (1989), Yamaguti and Prado (1995) and Veneziano, Moglin, and Bras (1995). The latter paper, in particular, emphasizes that the $f(\alpha)$ spectrum only generates the upper envelope of the true MFS, while missing interior points not in the upper envelope, and generates spurious points if the actual MFS is discrete (a bifractal would be an extreme example). We selected the canonical method of Chhabra and Jensen (1989), which is computationally efficient, avoids some of the problems faced by other techniques, and provides an intriguing mathematical connection to methods used in statistical mechanics. (See McCauley 1990 for a discussion of "thermodynamic formalism" for multifractals.)

The canonical method for computing $f(\alpha)$ begins by partitioning the image into boxes of width L. If $P_{i}(L)$ is the intensity integrated over box $i$, then $f(\alpha)$ is given implicitly by

$$
\begin{gathered}
f(q)=\lim _{L \rightarrow 0} \frac{\sum_{i} \mu_{i}(q) \log \mu_{i}(q)}{\log L} \\
\alpha(q)=\sum_{i} \mu_{i}(q) \alpha_{i}=\lim _{L \rightarrow 0} \frac{\sum_{i} \mu_{i}(q) \log P_{i}}{\log L}
\end{gathered}
$$

where $\mu_{i}(q)=P_{i}^{q}(L) / \sum_{i} P_{i}^{q}$ are the $q^{t h}$ coarse-grained moments of the original image and the sums cover the entire image. For real data sets, the limits are estimated by least square fitting of $\sum_{i} \mu_{i} \log \mu_{i}$ and $\sum_{i} \mu_{i} \log P_{i}$ as a function of $\log L$ for different $q$ values. If the linear fits are good and the slope varies with $q$, then the data set is said to possess multifractal scaling over the specified range of scales. If $f$ and $\alpha$ take on only a single value for all moments $q$, then the image is a homogeneous fractal.

The order parameter $q$ can be shown to be the slope of the $f(\alpha)$ curve, so the $f(\alpha)$ curve for multifractals is a convex downward opening curve peaking at the point where $q=0$. The value of $f$ at that point is the Hausdorff dimension of the support of the measure, which is 2 for the images considered here, while $\alpha(q=0)$ is just the average concentration strength of the image. At the point where $q=1$, the $f(\alpha)$ curve must lie on the diagonal line $f(\alpha)=\alpha$. The point at $q=\infty$ characterizes the regions in the image which have the smallest values of $\alpha$ which may be thought of as the sharpest spikes. Similarly, the $q=-\infty$ point characterizes the regions which have the steepest holes, i.e. with the largest values of $\alpha$. The $f(\alpha)$ function is related to the so-called generalized or Renyi dimensions $D_{q}$ (which characterize the scaling of the qth order partition function $\left.\sum_{i} P_{i}^{q}(L)\right)$ through a Legendre transform.

The dependence of the MFS on the parameters of a simple "toy model" multiplicative process is illustrated in Appendix B.

The referee has pointed out that the formalism involved in obtaining the MFS seems rather abstruse and non-intuitive, and suggests the general principle, with which we agree, that any empirical descriptor should bear a proportional requirement of success in terms of physical interpretation in exchange for complexity of effort. While we agree that the canonical formalism adopted here is not at all transparent, there are several points which suggest that the use of the MFS does not violate the above general principle. First, the computational complexity, if measured in cpu time, is actually smaller for the MFS than for the correlation function. Second, it must be 
recognized that the degree to which a descriptor seems obscure or complex depends inversely on the familiarity of the user. For example, the meaning and significance of the correlation function often seems obscure to those who have not dealt directly with it. Concerning the ability of the MFS to reveal some underlying physics, the reader may judge from the rest of the paper. We only remark that the MFS does allow us to infer the presence of some multiplicative process, and also shows a fundamental difference between incompressible and supersonic turbulence with respect to universality class, as discussed below. By comparison, studies of the velocity correlation function, for example, have revealed few significant clues to underlying physical processes, as far as we know.

\section{Results}

\subsection{Multifractal Scaling}

Figure 4 shows $\sum_{i} \mu_{i} \log \mu_{i}$ and $\sum_{i} \mu_{i} \log P_{i}$ as a function of $\log L$ for various positive $q$ values in the Taurus region. The range of box sizes used for the fits was 10 to 100 pixels, which corresponds to linear sizes of 0.4 to $4 \mathrm{pc}$. The lower limit is set by the resolution. For example, the adopted IRAS angular resolution of 3 arcmin corresponds to a linear resolution of $0.13 \mathrm{pc}$ for Taurus, at the assumed distance of $140 \mathrm{pc}$ (similar to all the regions studied here). Image structure is generally severely compromised for images smaller than 3-4 resolution elements, or $0.3-0.5 \mathrm{pc}$ for the regions studied here. The upper limit is set by the fact that at larger scales a significant fraction of the boxes used to compute the MFS overlap the edges of the images. We emphasize that the image size must be significantly larger than the upper limit for the range examined for scaling behavior; otherwise edge effects distort the scaling. Related edge effects give comparable distortion to other descriptors of structure (e.g. correlation function, see Houlahan and Scalo 1990 for an explicit demonstration). The panoramic nature of the IRAS images make it possible to satisfy this requirement. However, errors in calibration and background removal dominate and distort the scaling for negative $q$ values, which accentuate the low-flux areas. As a result, the scaling for negative $q$ values could not be investigated. The good linear fits (at least for lower $q$ values), with different slopes for different $q$ values, exhibited in Fig. 4 indicate that the column density structure in Taurus is multifractal, at least over the range of scales examined. These fits are typical of nearly all the subregions. Fig. 5 shows the scaling behavior of all of the subregions for a $q$ value of 8 . We conclude that all the regions used in this study exhibit multifractal scaling. This result demonstrates that there are no preferred scales in the column density structure over a factor of about ten in scale (roughly 0.4 to $4 \mathrm{pc}$ in the regions studied). Such a small range does not provide a very strong constraint on preferred scales, however.

Multifractal scaling is usually associated with multiplicative processes, involving hierarchical nesting or a "cascade." Meneveau and Chhabra (1990) and O'Brian and Chhabra (1992) have shown that the two-point correlation function of the spatial field of scaling exponents $\alpha, C_{\alpha}(s)$, should exhibit a logarithmic decline with separation $s$ for random isotropic multiplicative processes, providing a test for hierarchical structure. We have applied this test to the Taurus region by constructing an images of $\alpha$, and then calculating the correlation function $C_{\alpha}(s)$. The result is shown in Fig. 6, where it is seen that a significant range of logarithmic decay of $C_{\alpha}(s)$ exists. The fit to a logarithmic decay is even better than the turbulence examples shown in O'Brian and Chhabra (1992). We postpone a discussion of the other regions to a separate publication. 

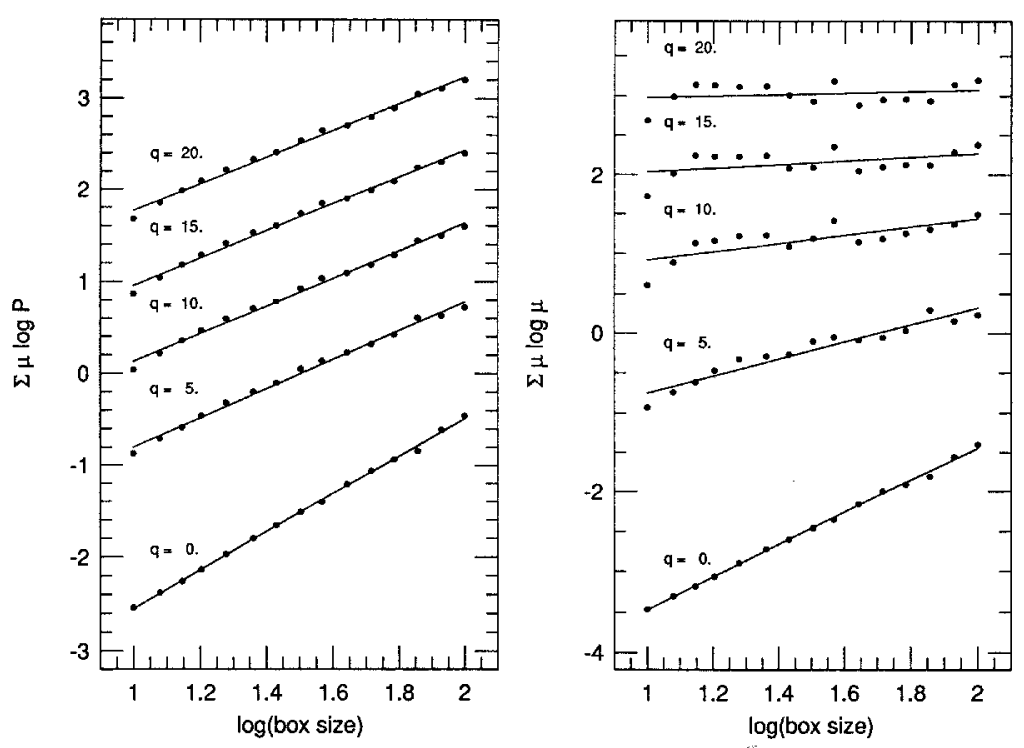

Fig. 4.- Multifractal scaling in Taurus. The slopes of the linear regression fits (indicated by straight lines) in the left panel gives the value of $\alpha$ for each $q$ value while the slopes in the right panel give the valves of $f$. The units of the abscissas are log arcminutes. At an assumed distance of $140 \mathrm{pc}$, the box size limits range from 0.4 to $4.0 \mathrm{pc}$. Arbitrary constants were added to each set of points to prevent the fits from overlapping.
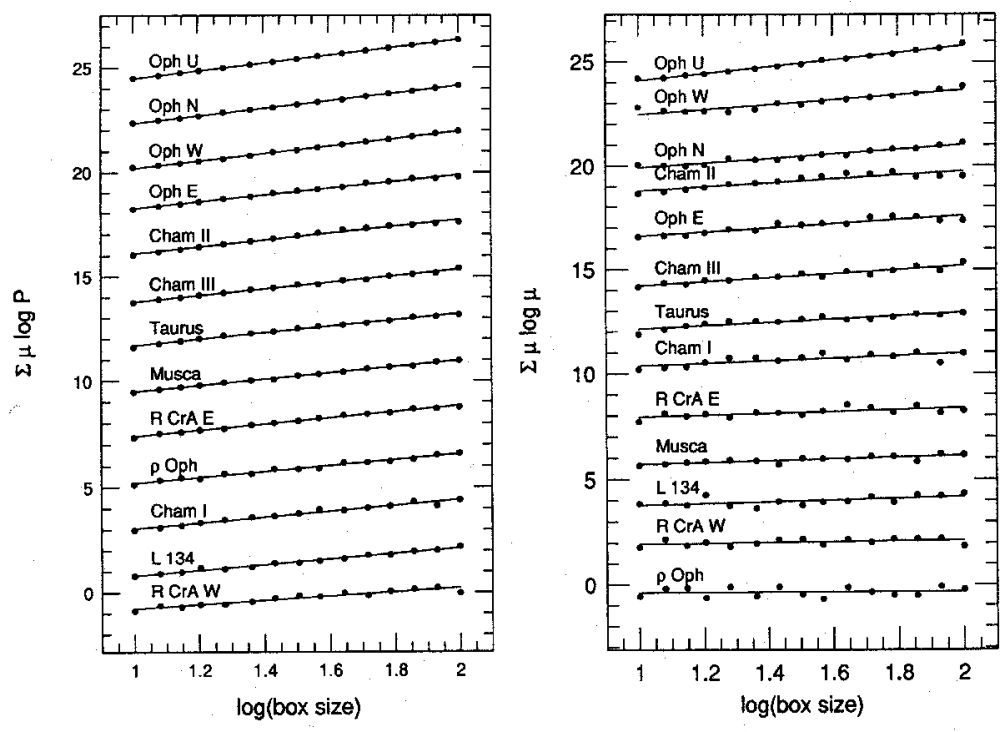

Fig. 5.- Multifractal scaling for all the regions. The fits for $\alpha$ and $f$ at $q=8$ are shown for all the regions. The fits are ordered by their slopes, with the steepest slopes at the top. Each set of data points were arbitrarily shifted up or down to prevent overlapping on the plot. 


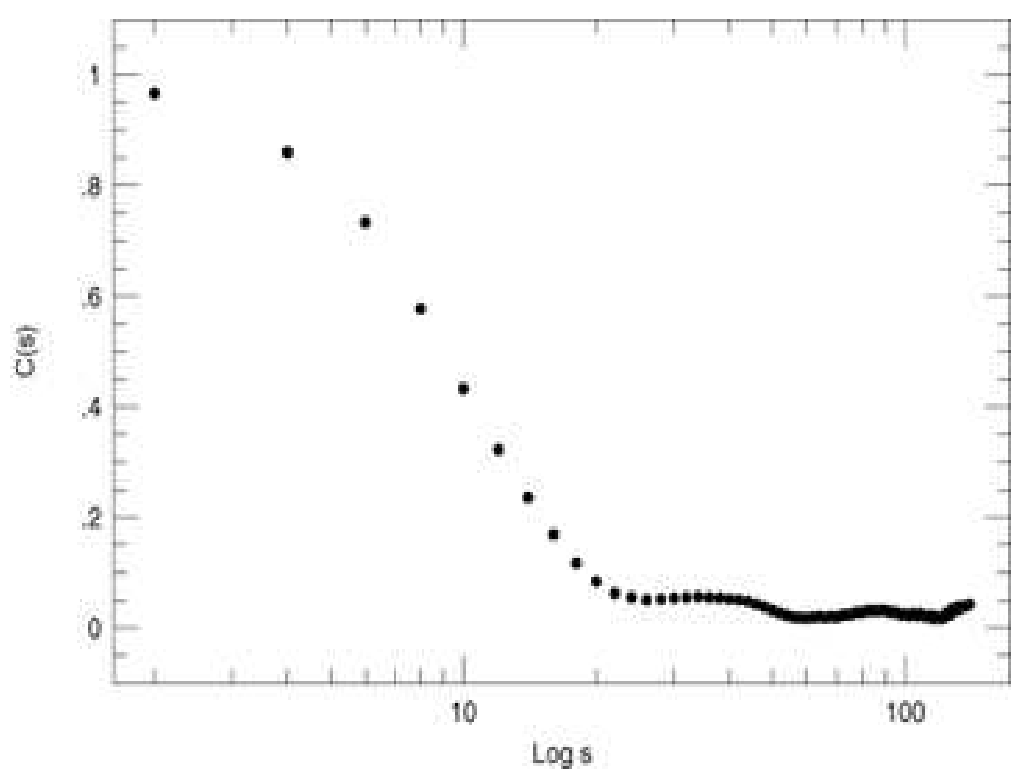

Fig. 6. - Correlation function for the image of concentration or "singularity" strengths $\alpha$ for the Taurus region. The significant range over which logarithmic decay occurs is interpreted as evidence for nested hierarchical structure (see text).

\subsection{Multifractal Spectrum}

Figure 7 shows the $f(\alpha)$ curve for positive $q$ values for each of the subregions. These $f(\alpha)$ curves were found to be fairly insensitive to the choice of background or to the adopted power law in the variation of dust emissivity with wavelength. The error bars represent the $\chi^{2}$ fitting error in determining $f$ and $\alpha$. The curves are ordered by their width, i.e. by $\alpha(q=20)$. The $f(\alpha)$ curves for the subregions display a variety of shapes.

We have attempted to determine whether the subregions could be uniquely ordered according to some overall property of the $f(\alpha)$ curves. Most of these attempts consisted of plotting the positions of the subregions in a two-dimensional diagram, each of whose axes correspond to some property of the $f(\alpha)$ curves. For example, one such property is the range of $f$ values covered by a fixed range of $q$ values which we chose as $4<q<20$ (since the $q<4$ points are very similar for most regions). This parameter $\Delta f \equiv f(q=4)-f(q=20)$ may be thought of as a "dimensional diversity," since images dominated by a single strong point-like concentration will have $f$ values close to zero for most positive $q$ values (low dimensional diversity), while those with a range of geometries (from surface-like to point-like) will have a large value of this parameter. Figure 8 shows the observed regions in the $\Delta f-\alpha(q=20)$ plane, where $\alpha(q=20)$ indicates the strength of the strongest concentrations. A fair correlation is present, but this is expected because the $f(\alpha)$ curve must, by its definition, remain below the diagonal line $f=\alpha$, so the range in $f$ values must decrease for curves which have smaller $\alpha$ values; i.e. images which possess strong dominant concentrations will usually have smaller diversities.

The ordering of the regions in Fig. 7 does correspond to the visual appearance of the geometry of the structures in the column density images. For example, R CrA W has the broadest $f(\alpha)$ and has the largest "point-like" contribution, consistent with the fact that the region is dominated by an extremely high-column density core. L134 and Cham I are also "point-like," although not as concentrated as R CrA W. At the other extreme, the $f(\alpha)$ curves for Oph N, W, and U are the 
narrowest of all the regions, in agreement with the smoother, diffuse, appearance and lack of strong concentrations seen in the column density images. The vertical extent of the $f(\alpha)$ curves for the most diffuse regions (Oph N,W,U) may be unreliable because of finite size effects. For example, it is well-known that a white noise field, which should appear as a single point at $\alpha=f=2$, will instead exhibit a very narrow measured $f(\alpha)$ that extends downward to smaller dimensions because of the finite size of the sample. Thus the dimensional diversity $f(q=4)-f(q=20)$ of Fig. 8 could be significantly smaller for these regions than calculated here. The intermediate regions (Musca, $\rho$ Oph, R CrA E, Cham II, Cham III, Taurus, and Oph E) all exhibit some quasi-linear or filamentary structure, mixed with point-like concentrations and more diffuse structures. Oph E does not contain any prominent filaments, being primarily diffuse like Oph N,W,U, but it does contain an irregular "ridge" that may reflect the presence of an ionization/shock front, and it is probably this feature that shifts the $f(\alpha)$ spectrum toward the regions with prominent filaments. Notice also that the total $\mathrm{R}$ CrA region visually resembles the $\rho$ Oph region dense core with "streamers" to one side. It is because we have divided R CrA into two subregions (the core and the streamers) that the core, R CrA W, separates so clearly from the rest of the regions in Fig. 8.

This result illustrates the problematic dependence of all structural descriptors on the subjective pre-selection of the region to be described.
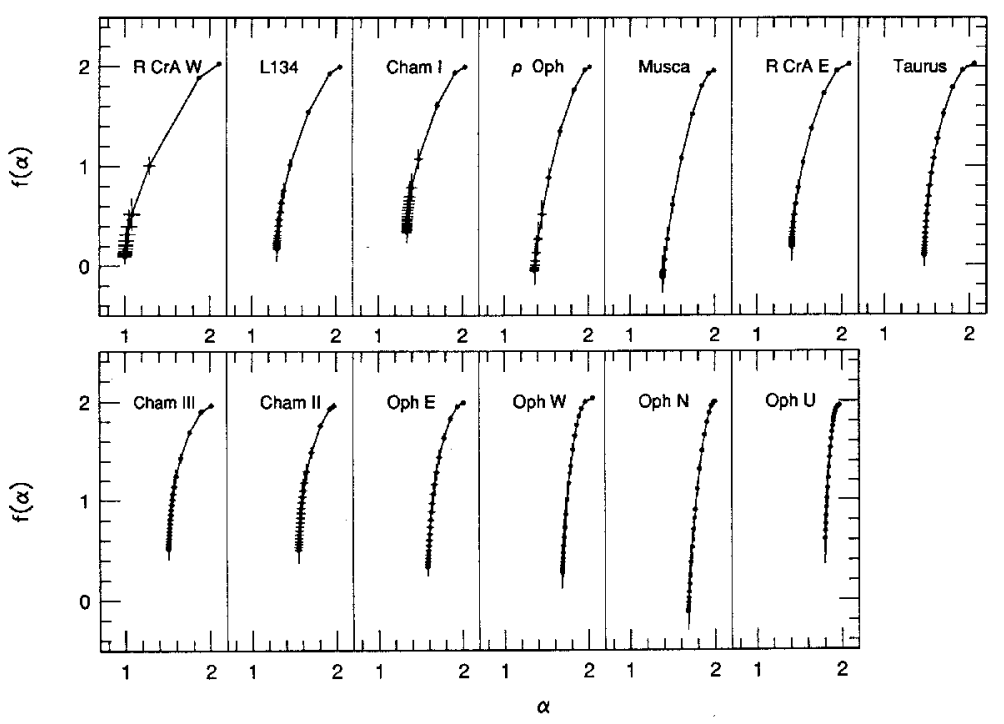

Fig. 7.- Spectrum of singularities of the column density maps of all the subregions. Due to background and calibration uncertainties only the left positive- $q$ portion of the $f(\alpha)$ curves were calculated. The regions are ordered by the strength of the strongest singularities present, i.e. by $\alpha(q=20)$ which is the width of the $f(\alpha)$ curve. 


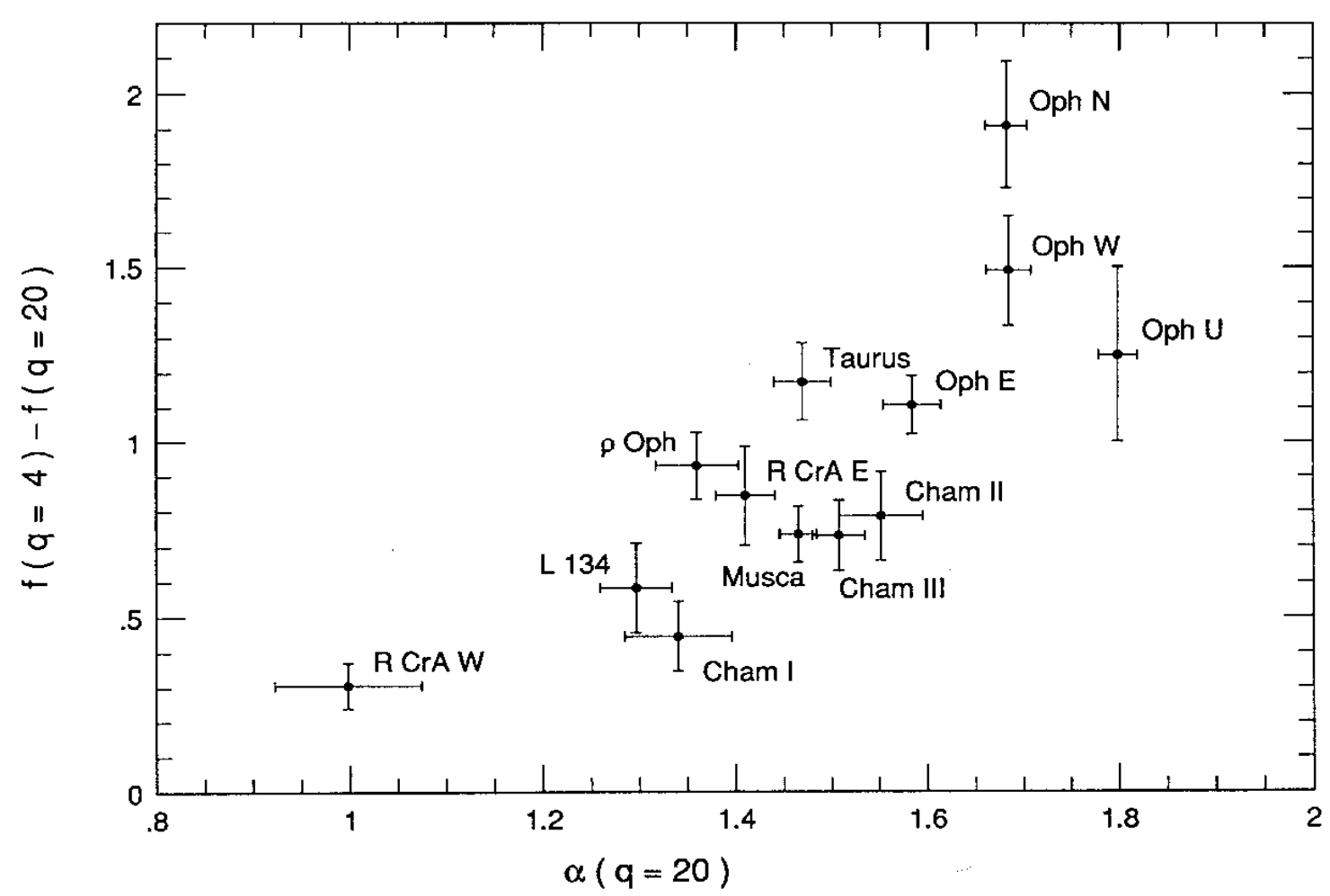

Fig. 8. - The "dimensional diversity," taken as $f(q=4)-f(q=20)$, is plotted as a function of the strength of the strongest singularities present in the region, i.e. $\alpha(q=20)$.

Since the ordering of regions described above does involve the degree of concentration, it might be erroneously thought that the properties of $f(\alpha)$ at large $q$ are simply reflecting the degree of density contrast. We have examined the probability distributions of column density for all the subregions and their moments. The standard deviation (s.d.) relative to the mean column density varies from 0.20 (Musca) to 0.55 ( $\mathrm{R}$ CrA W). So R CrA W is extreme in both $f(\alpha)$ ordering and column density contrast, and, similarly, Oph U and Oph $\mathrm{N}$ are rather extreme at the other end, ranking 10 and 8 (out of 11) in standard deviation, respectively. However there are exceptions that make it clear that the $f(\alpha)$ ranking is primarily geometrical, even if density contrast does correlate with geometry in some cases. For example, Musca has by far the lowest s.d., yet lies in the middle of the regions in Fig. 9 because it is primarily a simple filament. L 134 is ranked 7 in s.d. (0.34), yet it, along with Cham I, is, after R CrA W, the most "point-like" of the regions, not at all like Oph N, which has a similar s.d. Another example is R CrA E, which is ranked 2 in s.d. (0.46), even though it is near Musca (rank 11), Cham II (rank 9), and Cham III (rank 6) in Fig. 8 due to the prevalence of line-like structure in all these regions. Oph $\mathrm{W}$ is among the three most "space-filling" or homogeneous regions according to Fig. 8, yet its standard deviation is rank 5 (0.38). Clearly the $f(\alpha)$ indicator is responding to geometry and not density contrast in these cases.

It should be emphasized that, while some properties of $f(\alpha)$ do reflect some of the gross character of the geometry of these regions, and hence may be useful descriptors for classification and comparison with simulations, $f(\alpha)$ definitely does not capture other aspects of structural detail. For example, the Cham II and Cham III regions have very similar $f(\alpha)$ curves but different visual appearances: The Cham III region looks more "disrupted" or filamentary (even though it contains few, if any, young stellar objects; see below). Thus, the $f(\alpha)$ spectrum, like all other 
structural descriptors that have been proposed, suffers from severe compression of structural information. On the other hand, the utility of different structural descriptors, even if, or just because, they are highly compressed, is their ability to capture different aspects of the structure, and in that regard the geometrical information expressed compactly by $f(\alpha)$ is important because those aspects are not expressed by other descriptors. However it appears that no quantitative descriptor will be capable of capturing any but a small fraction of the aspects of structure apparent to the eye/brain. This is of course a common and severe problem in all applications of pattern analysis when description, rather than specific recognition or identification, is the goal.

Our method of ordering the regions in terms of some property of the $f(\alpha)$ curve is not unique, and we considered other approaches. For example, we tried to order the regions according to the distance of $f(\alpha)$ from some fiducial reference $f(\alpha)$ curve, defining distance as in Adams (1992) and Adams and Wiseman (1994). For the fiducial curve we tried various colored noises, stochastic multiplicative processes, and the mean $f(\alpha)$ for the observed region. However it was found that the resulting ordering depended strongly on the choice of the standard $f(\alpha)$ curve. We suspect that this dependence of ordering on the adopted reference function will be a general problem for this approach to classification. Instead we would suggest that the variation of ordering with respect to fiducial reference function is itself an important descriptor of complexity, since it is an indication of the number of ways in which the collection of regions can be viewed; i.e. a measure of the ambiguity presented by the structure. This has been a common theme in recent discussions of the meaning of complexity, although we know of no quantitative measure that has been proposed.

The relation of $f(\alpha)$ to the stellar content of the different regions is also of interest. It is generally thought that the star formation properties of cloud complexes might be related to the geometry of the dust and gas, although even the direction of the causation is unclear. Larson (1992) proposed that the stellar initial mass function may be a consequence of the geometry of the gas from which stars form. From a different point of view, it is generally thought that the presence of young stars should alter the structure of the gas in the parent cloud complex in a severe manner, although no quantitative descriptor of this effect has, to our knowledge, been proposed. For this reason, several star formation indicators were compiled for each of the regions, including the surface density of young stellar objects, the ratio of stellar luminosity to gas mass, and the star formation efficiency. (See the references in Table 1; also the study of star formation in these regions by Chen et al. 1997.) No correlation with the shape of the $f(\alpha)$ curve was found. The reason for this lack of correlation is fairly obvious: There is simply no one-to-one correspondence between gas morphology and stellar content. For example, while L134, with little internal activity, has a much smaller column density than either $\rho$ Oph or R CrA W, it certainly does have a "core-like" appearance, and so is geometrically similar to the cores with strong star formation activity. When discussing "cores", this lack of correspondence may seem trivial, since it might simply be due to the fact that a region without internal activity hasn't had time to begin star formation, or has too small a mass to be bound, etc. However other examples show that the lack of correspondence goes further than regions that appear to comply with conceptions of quasi-spherical "clouds." Both Taurus and Cham III have similarly prominent filamentary structure, but Cham III is devoid of star formation activity. Musca is another filament with little internal activity; the same applies to the streamers extending away from the $\rho$ Oph and R CrA cores. The differences may be due to the ages of the regions or because the gas morphology reflects external forces (particularly for Musca) as well as internal processes like self-gravity or hydrodynamic instabilities. Perhaps the most interesting comparison is Cham II and III. As mentioned above, Cham III appears significantly more "disrupted," even though it has essentially no measurable internal star formation activity. These examples suggest that it will be quite difficult to understand any relationship between star formation and gas structure, given the small number of regions that can be mapped at sufficient resolution, and the fact that we observe each region at different effective ages. Still, it would be interesting to carry out the type of geometrical analysis presented here for the column density structures in regions with strong massive star formation. The IRAS data are unsuitable for such maps because the heating by the massive stars probably seriously distorts the derived column densities, as explained in sec.II. The Bell Labs ${ }^{13} \mathrm{CO}$ survey provides sufficiently dense mapping of massive star formation regions for estimates of $f(\alpha)$ (for the column densities and the radial velocities), and should be attempted. Based on the results found here, it is likely that $f(\alpha)$ will 
primarily reflect the mix of strong "point-like" structures (very dense "cores") and filamentary structures, both of which are known to exist in these regions. Thus, there is no reason to expect the properties of the $f(\alpha)$ curve to be significantly different from those found for, say, Taurus or $\rho$ Oph.

\section{Summary}

We have investigated the possibility of multifractal scaling in several regions and subregions of local cloud complexes using column density images with a very large spatial dynamic range constructed from IRAS data. The major results are as follows.

1. All the regions and subregions exhibited multifractal scaling over the range 0.4 to 4 parsecs, the largest range available for investigating scaling using the available images and this technique, even though the images covered a much larger range, because of the sensitivity to finite size and resolution effects (see also Takayasu and Suzuki 1991). That the column density images do exhibit multifractal power law scaling is a new and surprising result, which should provide an important constraint on theoretical models for the physical origin of the structure in star-forming clouds. In particular, since MFS is commonly associated with multiplicative processes, the existence of MFS in cloud complexes can be taken as indirect evidence for nested hierarchical structure. We have shown that the correlation function of the $\alpha$-images of Taurus exhibits a distinct range in which logarithmic decay occurs, consistent with predictions for a hierarchical multifractal process.

2. Contrary to results obtained for the dissipation fields and passive scalar fields of incompressible turbulence (Sreenivasan 1996 and references therein; also Muzy et al. 1991), the $f(\alpha)$ curves for these interstellar regions have a variety of shapes indicating that they do not belong to a single universality class with respect to $f(\alpha)$. This result suggests to us that supersonic magneto-gravitational "turbulence" does not possess a statistical equilibrium range, or that any such quasi-equilibrium evolves significantly with time or depends on initial or environmental conditions. This is in contrast to the similar perimeter-area dimension found in previous studies of local star-forming cloud complexes. However it remains to be seen whether the radial velocity images of these regions are multifractal, and whether they exhibit a universal MFS.

3. The forms of the $f(\alpha)$ curves for these regions do seem to correlate fairly well with the mixture of geometric forms seen visually. Thus the MFS appears to be a useful descriptor for capturing this aspect of structure, and should be useful in combination with other descriptors in eventually obtaining a quantitative classification scheme for interstellar structure. However we find no correlation between the forms of the $f(\alpha)$ curves and the level of internal star formation activity. This lack of correlation between morphological structure and star formation activity can be easily seen directly in several examples by eye.

The fact that all the regions do exhibit well-defined multifractal scaling provides a new constraint on theoretical models for cloud evolution and star formation. Simulations of realistic ISM evolution are approaching sufficient resolution to allow a search for multifractal scaling, although no such search has yet been conducted. It is known that simple models for both hierarchical fragmentation (e.g. sequential probabilistic partitioning, as has been used to model the $f(\alpha)$ spectrum of incompressible turbulence) and coalescence (aggregation) can result in multifractal scaling. However, in the fragmentation case the dominant physics is unclear, since the structure might be produced by nonlinear fluid advection, as in incompressible turbulence, or by repeated gravitational instability or other fluid instabilities. In the case of coalescence, multifractal scaling has only been demonstrated for Monte Carlo simulations in which spatial correlations were built in using a specific power law correlation function (Menci et al. 1993). It is hoped that the present results will motivate theoretical searches for multifractal scaling in simulations of cloud evolution based on the hydrodynamic equations. Although very large spatial dynamic range is required, even a negative result would allow one to conclude that an important physical process has been neglected, while a positive result might allow an identification of the physical processes that are responsible for the variation in $f(\alpha)$ found among different star-forming regions. 
We thank the referee, whose comments on an earlier form of this paper led to considerable re-thinking and improvement in the presentation. We are grateful to Mark Heyer for providing the digitized ${ }^{13} \mathrm{CO}$ data for Taurus, Chris Brunt for useful correspondence, and Fran Verter for helpful comments concerning the treatment of the IRAS data. This work was supported by NASA grant NAG5-3107 and a grant from Texas Advanced Research Projects.

\section{A. Validity of IRAS Column Densities}

In order to understand the degree to which the IRAS $100 \mu \mathrm{m}$ column densities, $\mathrm{N}_{100}$, represent the true column density distribution, we have compared our $\mathrm{N}_{100}$ images to images of five subregions in Taurus constructed from ${ }^{13} \mathrm{CO}$ (Heyer et al. 1987), visual extinction $\mathrm{A}_{\mathrm{V}}$ (Cernicharo and Bachillar 1984), and, in the case of L1495, $\mathrm{C}^{18} \mathrm{O}$ (Duvert et al. 1986). These subregions are L1529(=B18), B217, L1495, L1536, and Heiles Cloud 2. Both ${ }^{13} \mathrm{CO}$ and $\mathrm{A}_{\mathrm{V}}$ were available for Heiles Cloud 2 and L1529, while only ${ }^{13} \mathrm{CO}$ was available for B217 and only $\mathrm{A}_{\mathrm{V}}$ for L1506, L1495, and L1536.

A large-scale logarithmically scaled $\mathrm{N}_{100}$ image of Taurus is presented in Figure 9. The image has been processed by unsharp masking to bring out the smaller-scale structure. The unsharp masking is equivalent to artificially modifying the column density power spectrum by putting more power at larger wavenumbers. We were careful to verify that this image enhancement did not introduce any spurious or artifactual features. The calculations in the text and the comparisons described below are based on unprocessed images.

The comparisons all yielded surprisingly positive results in the sense that the resulting structures are very similar in visual appearance. A comparison of ${ }^{13} \mathrm{CO}, \mathrm{N}_{100}$, and $\mathrm{A}_{\mathrm{V}}$ for the L1529 subregion (the filament in the lower center of Fig. 9) is presented in Figure 10. The ${ }^{13} \mathrm{CO}$ and $\mathrm{A}_{\mathrm{V}}$ images were taken in digitized form directly from the original data, but have been smoothed to the adopted resolution of the IRAS data. The agreement in morphology is good, especially compared to the ${ }^{13} \mathrm{CO}$ data. Notice the nearly circular ring extending from the middle northern part of this substructure, which can be seen to various degrees in all three maps, although its ring-like form is barely discernable in the extinction image. We concur with Heyer et al. 's conclusion that the extinction errors must be considerably larger than usually quoted in the literature.

Fig. 10 is typical of the five comparisons that were carried out. The only discrepancy was found for the very opaque core of L1495. Luckily, a $\mathrm{C}^{18} \mathrm{O}$ map was available for this region (Duvert et al. 1986), which showed that the "extra structure" seen in the IRAS map does indeed appear similarly in the $\mathrm{C}^{18} \mathrm{O}$ map; the agreement is only compromised by the presence of an embedded source which produces a spurious "hole" in the IRAS map-otherwise the agreement is surprisingly good. We conclude that the ${ }^{13} \mathrm{CO}$ and $\mathrm{A}_{\mathrm{V}}$ maps are saturated, and that the IRAS $100 \mu \mathrm{m}$ column density map probes optical depths similar to that probed by $\mathrm{C}^{18} \mathrm{O}$, at least in this case.

Another indication that the IRAS column density maps presented here faithfully represent the structure is the excellent agreement between our images and the adaptive grid/wavelet transform optical extinction maps presented by Cambresy (1999) for Taurus, Sco-Oph, R Cr A, and Cham-Musca. The techniques used by Cambresy allow extinction to probe much larger column densities, and more accurately, than previous extinction studies. 


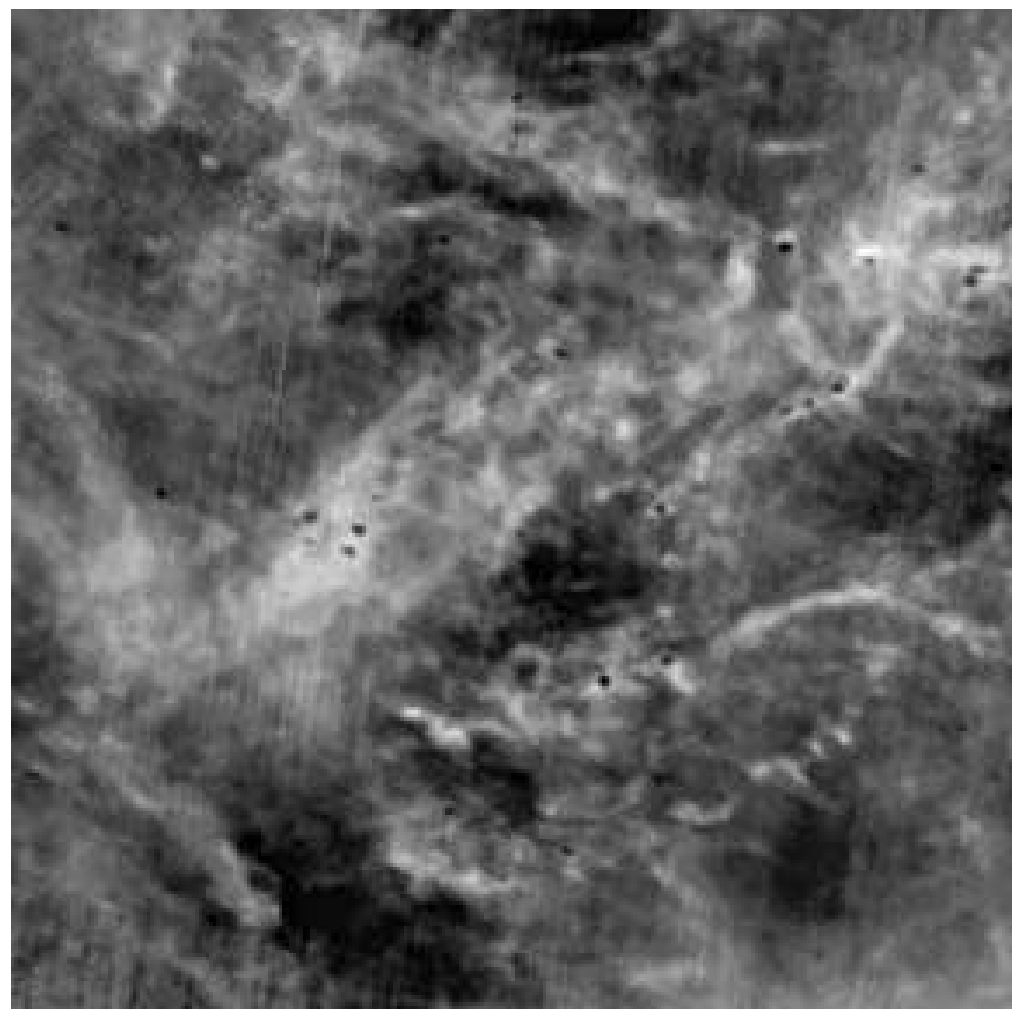

Fig. 9.- $100 \mu \mathrm{m}$ column density map of Taurus, with unsharp masking applied to enhance small scale structure. 

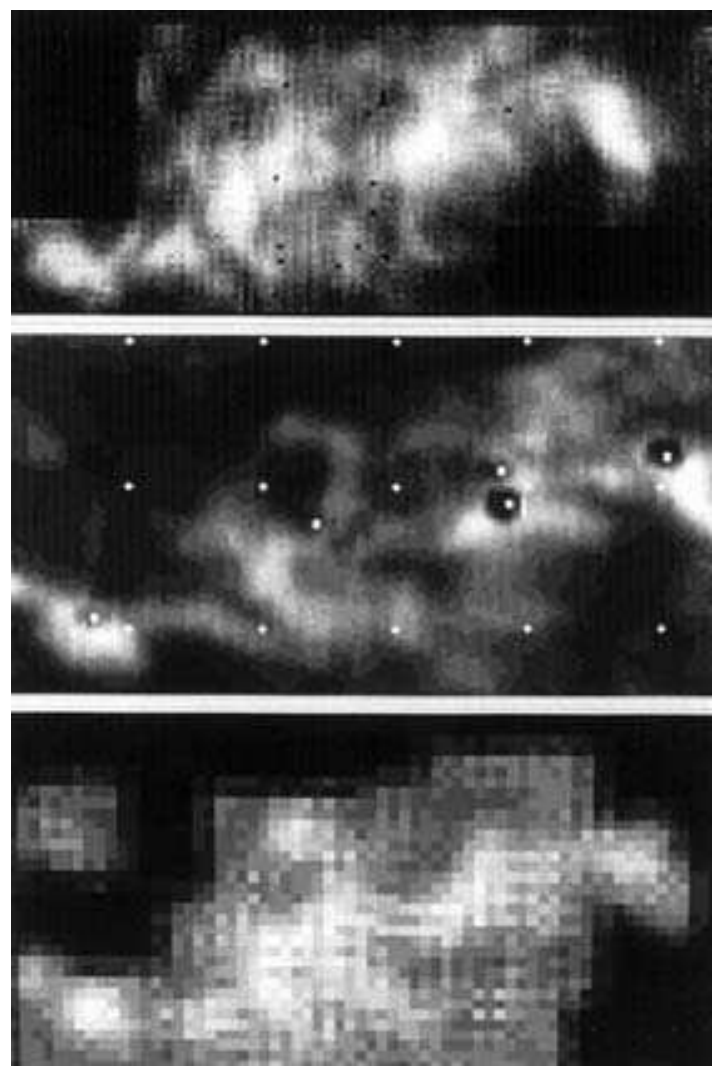

Fig. 10.- Comparison of column density images for L1529 in Taurus based on ${ }^{13} \mathrm{CO}$ (top), $\mathrm{N}_{100}$ (middle), and $\mathrm{A}_{\mathrm{V}}$ (bottom).

\section{B. Multiplicative Toy Models}

The $f(\alpha)$ spectrum is ideally suited to characterizing structure which can be created by a multiplicative process. We present one such process, called the p-model (see Sreenivasan 1991) or multinomial multiplicative process (Feder 1988), to demonstrate the sensitivity of the $f(\alpha)$ spectrum to both scaling and geometry in images. 

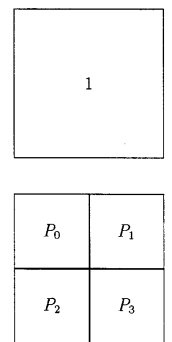

$$
\begin{array}{|l|l|l|l|}
\hline P_{0} P_{0} & P_{0} P_{1} & P_{1} P_{0} & P_{1} P_{1} \\
\hline P_{0} P_{2} & P_{0} P_{3} & P_{1} P_{2} & P_{1} P_{3} \\
\hline P_{2} P_{0} & P_{2} P_{1} & P_{3} P_{0} & P_{3} P_{1} \\
\hline P_{2} P_{2} & P_{2} P_{3} & P_{3} P_{2} & P_{3} P_{3} \\
\hline
\end{array}
$$

$\ldots$

Fig. 11. - The multiplicative construction process defining the toy models. A square with an initially uniform measure is divided into four smaller squares. In each, the original measure is multiplied by a constant $P_{i}$, where $i \epsilon\{0,1,2,3\}$. The set $\left\{P_{i}\right\}$ remains fixed throughout the entire construction process. Each of the squares are then divided once again, and the construction process is repeated a large number of times. The final function will consist of a large number of spikes and is known to possess multifractal scaling. This is Sreenivisan's (1991) "p-model." The $f(\alpha)$ curves for several multifractals generated with the p-model are shown in Fig. 12.

Figure 11 shows the construction process for the model. A uniform square is divided into four smaller squares each receiving a probability $p_{\mathrm{i}}$. Each smaller square, in turn, is subdivided into still smaller squares, the probability in each given by the product of $p_{\text {i }}$ with the probability of the "parent" square. Iterating this process produces a probability distribution densely filled with singularities. Singularities with common exponents, $\alpha$, are distributed as homogeneous fractals; however the fractal dimension varies as a function of the exponent (Feder 1988). The $f(\alpha)$ spectrum provides a convenient method of characterizing both the scaling exponents of the singularities and the fractal dimension of the "iso- $\alpha$ " sets. 


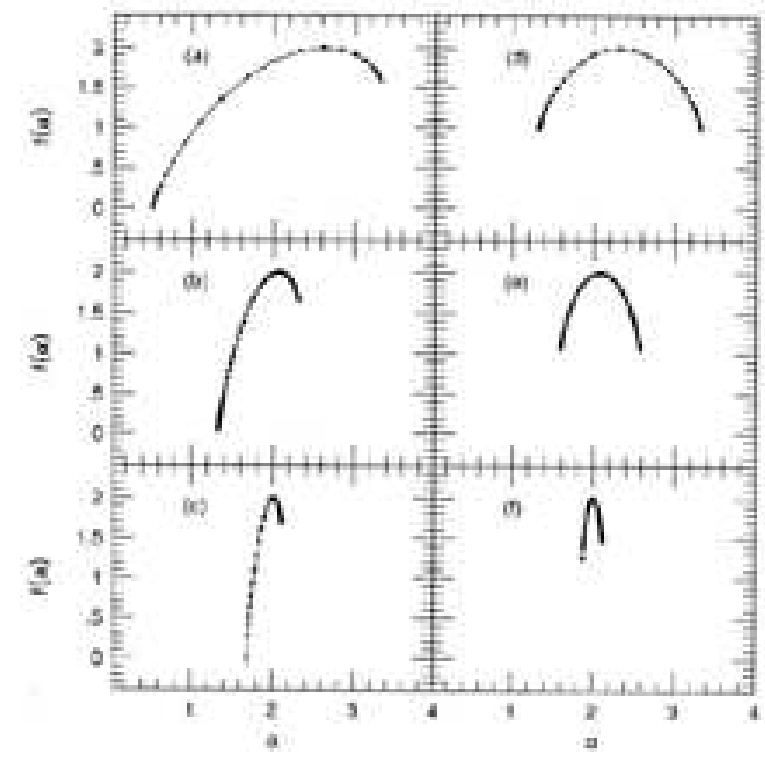

Fig. 12.- The $f(\alpha)$ singularity spectrum for the toy multiplicative models defined in Fig. 11. In the right-hand panels the diagonal probabilities are set equal $P_{1}=P_{2}$ and $P_{0}=P_{3} \equiv 0.5-P_{1}$. In the left-hand panels three probabilities are set equal $P_{1}=P_{2}=P_{3}$ with $P_{0} \equiv 1-3 P_{1}$. In each case, the difference $\left|P_{1}-P_{0}\right|$ increases up the page producing stronger "singularities."

Figure 12 shows the $f(\alpha)$ spectrum for several p-models. In each case the pattern of probabilities was kept constant for all stages of the construction. In the left hand column, the largest probability is given by $p_{1}$ and the remaining three probabilities are set equal $p_{2}=p_{3}=p_{4} \equiv\left(1-p_{1}\right) / 3$. The ratio $p_{1} / p_{2}$ is decreased down the column. All the curves peak at $f(q=0)=2$ corresponding to the two-dimensionality of the images. The asymmetry of the $f(\alpha)$ curves reflects the asymmetry in the spatial distributions of the strong and weak singularities; the strongest singularities are distributed as isolated points $(f(q=\infty)=0)$, whereas the weakest singularities are more densely distributed and can be shown to lie on a "Serpinski gasket" with a fractal dimension $f(q=-\infty) \approx 1.58$.

The value of $\alpha$ at the $q=\infty$ point represents the strength of the strongest singularities and decreases as the ratio $p_{1} / p_{2}$ increases. The width of the $f(\alpha)$ curve measures the range of the singularity strengths present in the image.

The curves in the right-hand column are based on p-models in which $p_{1}=p_{3}$ and $p_{2}=p_{4} \equiv\left(1-p_{1}\right) / 2$. Again, the ratio $p_{1} / p_{2}$ is decreased down the column. The strongest singularities occur where the probability distribution is largest, i.e. where the probabilities $p_{1}$ or $p_{3}$ have been used in each stage of the construction process. Since the number of such squares at the $n^{\text {th }}$ construction stage scales as $n \propto L^{-1}$, where $L$ is the box size, the strongest singularities have a fractal dimension $D=1$. Similarly, the weakest singularities also have $D=1$, producing a symmetrical $f(\alpha)$ curve which remains above $f=1$.

We conclude with a cautionary remark. Figure 13 shows three point sets which evoke different visual impressions yet which possess the same fractal dimension of $D=1.0$. It is obvious that the fractal dimension (and therefore the $f(\alpha)$ spectrum) is a severely incomplete and degenerate description of the underlying structure and care should be taken when making inferences based on this (or any other) structural descriptor. A well-known similar example is the power spectrum (and hence correlation function), which is highly degenerate (very dissimilar structures can produce nearly-identical power spectra) because phase information is lost in its construction. 
More detailed discussion of the ambiguity involved in interpreting the multifractal spectrum can be found in Chhabra, Jensen, and Sreenivasan (1989) and Bulajich and Perez-Pascual (1991).

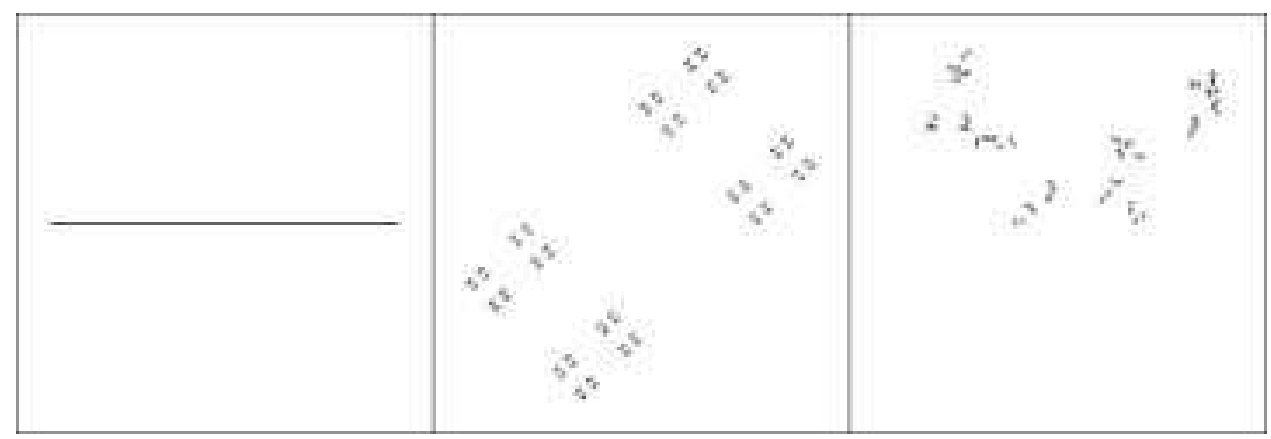

Fig. 13. - Examples showing the degeneracy of the fractal dimension as a structure descriptor. Each point set has a fractal dimension $D=1$. The fractal in the center panel was created with the fixed p-model with $P_{1}=P_{2}=0.5$ and $P_{0}=P_{3}=0$ (see Fig. 11). The ordering of the probabilities was reversed on alternate stages of the construction process. The fractal in the right-hand panel was constructed using the same set of probabilities, but with random ordering at each stage of the construction process. 


\section{References}

Abergel, A., Boulanger, F., Fukuii, Y., and Mizuno, A. 1995, A\&A Supp., 111, 483

Abergel, A., Boulanger, F., Mizuno, A., and Fukui, Y. 1994, ApJL, 423, L59.

Adams, F. C. 1992, ApJ, 387, 572

Adams, F. C. and Wiseman, J. J. 1994, ApJ, 435, 693

Arce, H. G. and Goodman, A. A. 1999, ApJ, 517, 264.

Balafas, J. S. and Dewey, T. G. 1995, Phys. Rev. E, 52, 880

Bally, J. 1989, in Structure and Dynamics of the Interstellar Medium, IAU Colloquium 120, ed. G. Tenorio-Tagle, M. Moles, and J. Melnick (Springer: Berlin), p. 309

Bally, J., Langer, W. D., Stark, A. A., and Wilson, R. W. 1987, ApJL, 312, L45

Baud, B., Young, E., Beichman, C. A., et al. 1984, ApJ, 278, L53

Bazell, D. and Desert, F. X. 1988, ApJ, 333, 353

Beckwith, S.V.W., Sargent, A. I., Chini, R. S., and Gusten, R. 1990, AJ, 99, 924

Beech, M. 1987, Ap. Sp. Sci., 133, 193

Bhavsar, S.P. and Ling, E.N. 1988, ApJL, 331, L63

Bulajich, R. and Pérez-Pascual, R. 1991, in Nonlinear Phenomena in Fluids, Solids and Other Complex Systems, ed. P. Cordero and B. Nachtergaele (Elsevier Sci. Publ.), p. 435

Cambresy, L. 1999, A\&A, 345, 965

Cernicharo, J. 1991, in The Physics of Star Formation and Early Stellar Evolution, ed C. J. Lada and N. D. Kylafis (Dordrecht: Kluwer), p. 287

Cernicharo, J. and Bachillar, R. 1984, A\&A Suppl, 58, 327

Chhabra, A. and Jensen, R. V. 1989, Phys. Rev. Lett., 62, 1327

Chhabra, A.B., Jensen, R. V., and Sreenivasan, K. R. 1989, Phys. Rev. A, 40, 4593

Chhabra, A.B. and Sreenivasan, K.R. 1992, Phys. Rev. Lett., 68, 2762

Chen, H., Grenfell, T. G., Myers, P. C., and Hughes, J. D. 1997, ApJ, 478, 295

Colombi, S., Bouchet, F. R., Schaeffer, R. 1992, A\&A, 263, 1

DeGeus, E. J., de Zeew, P. T., Lub, J. 1989, A\&A, 216, 44

Dickman, R. L., Horvath, M. A., and Margulis, M. 1990, ApJ, 365, 586

Duvert, G., Cernicharo, J., and Baudry, A. 1986, A\&A, 164, 349

Elias, J. H. 1978, ApJ, 224, 857

Elmegreen, B. G. 1992 in The Galactic Interstellar Medium, SAAS FEE Advanced Course 21, ed. D. Pfenniger and P. Bartholdi, pp. 157-274

Elmegreen, B. G. 1997, ApJ, 477, 196 
Elmegreen, B. G. and Falgarone, E. 1996, ApJ, 471,816

Falgarone, E., Puget, J.-L., and Perault, M. 1992, A\&A, 257, 715

Falgarone, E., and Phillips, T. G. 1991, in Fragmentation of Molecular Clouds and Star Formation, ed. E. Falgarone, F. Boulanger, and G. Duvert (Dordrecht: Reidel), p. 119

Falgarone, E., Phillips, T. G., and Walker, C. K. 1991, ApJ, 378, 186

Feder, J. 1988, Fractals (New York: Plenum Press)

Franco, G.A.P. 1991, A\&A, 251, 581

Genzel, R. 1991, in Molecular Clouds, ed R. A. James and T. J. Millar (Cambridge: Cambridge Univ. Press), p. 75

Graham, J. A. 1990, in Low Mass Star Formation in Southern Molecular Clouds, ed. B. Reipurth $(\mathrm{ESO})$

Gregorio-Hetem, J., Sanzovo, G. C., and Lepine, J.R.D. 1988, A\&A, 76, 347

Henriksen, R. N. 1991, ApJ, 377, 500

Hetem, A. and Lépine, J.R.D. 1993, A\&A, 270, 451

Heyer, M. H., Vrba, F. J., Snell, R. L., Schloerb, F. P., Strom, S. E., Goldsmith, P. F., and Strom, K. M. 1987, ApJ, 312, 855

Hosokawa, I. and Yamamoto, K. 1990, Phys. Fluids A, 2, 889

Houlahan, P. and Scalo, J. M. 1990, ApJ Suppl., 72, 133

Houlahan, P. and Scalo, J.M. 1992, ApJ, 393, 172.

Jarrett, T. H., Dickman, R. L., and Herbst, W. 1989, ApJ, 345, 881

Kawamura, A., Onishi, T., Yonekura, Y., Dobashi, K., Mizumo, A., Ogawa, H., \& Fukui, Y. 1999, in Star Formation 1999, ed. T. Nakamoto (Nobeyama Radio Observatory), p. 76

Kenyon, S. J., Hartmann, L. W., Strom, K. M., and Strom, S. E. 1990, AJ, 99, 869

Lada, C. J., Alves, J., and lada, E. A. 1999, ApJ, 512, 250

Langer, W. D., Wilson, R. W., Goldsmith, P. F., and Beichman, L. A. 1989, ApJ, 337, 739

Larson, R. 1992, MNRAS, 256, 641

Lawrence, J. K., Ruzmaikin, A. A., and Cadavid, A. C. 1993, ApJ, 417, 805

Lovejoy, S. 1982, Science, 216, 185

Luo, S., Vishniac, E.T., and Martel, H. 1996, ApJ, 468, 62

Marraco, H. G. and Rydgren, A. E. 1981, ApJ, 86, 62

Martinez, V. J., Jones, B.J.T., Dominguez-Tenreiro, R., and van de Weygaert, R. 1990, ApJ, 357, 50

McCauley, J. L. 1990, Phys. Reports, 189, 225

Menci, N., Colafrancesco, S., and Biferale, L. 1993, J. de Physique I, 3, 1105 
Meneveau, C. and Chhabra, A. B. 1990, Physica A, 164, 564

Meneveau, C. and Sreenivasan, K. R. 1989, Phys. Rev. Lett., 59, 1424

Mott, F., Andre, P., and Neri, R. 1998, A\&A, 336, 150

Muller, J. Rambak, J. P., Clausen, O. P. F., and Hovig, T. 1991, in Complexity, Chaos, and Biological Evolution, eds. E. Mosekilde and L. Mosekilde (NY: Plenem Press), p. 377

Muzy, J. F., Bacry, E., and Arneodo, A. 1991, Phys. Rev. Lett., 67, 3515

Muzzio, F. J., Meneveaus, C., Swanson, P. D., and Ottino, J. M. 1992, Phys. Fluids. A, 7, 1439

Nagatani, T. 1992, J. Phys. A, 25, L955

O'Brian and Chhabra, A. B. 1992, preprint.

Rossano, G. S. 1978a, AJ, 83, 234

$$
\text { 1978b, AJ, 83, } 241
$$

Sargent, A. I., van Duinen, R. J., Nordh, H. L., Fridlund, G.V.M., Aalders, J.W.G., and Beintema, D. 1983, AJ, 88,88

Scalo, J. M. 1985, in Protostars and Planets II, eds. D. C. Black and M. S. Mathews (Tucson: Univ. of Arizona Press), p. 201

Scalo, J. M. 1990, in Physical Processes in Fragmentation and Star Formation, ed. R. Capuzzo-Dolcetta et al. (Dordrecht: Reidel), p. 151

Schwartz, R. D. 1989, in Low Mass Star Formation in Southern Molecular Clouds, ed. B. Reipurth (ESO)

Snell, R. L. 1981, ApJ, 45, 121

Snell, R. L., Schoerb, F. P., Heyer, M. H. 1989, ApJ, 337, 739

Sreenivasan, K. R. 1991, Ann. Rev. Fluid Mech., 23, 539

Sreenivasan, K. R. 1996, in Research Trends in Fluid Dynamics, eds. J. L. Lumley, A. Acrivos, L. G. Leal, and S. Leibovich (Woodbury, N.Y.: AIP Press), p. 263

Stutzki, J., Genzel, R., Graf, U., Harris, A., Sternberg, A., and Gusten, R. 1991, in Fragmentation of Molecular Clouds and Star Formation, ed. E. Falgarone, F. Boulanger, and G. Duvert (Dordrecht: Reidel), p. 235

Sylos Labini, F. and Pietronero, L. 1996, ApJ, 469, 26

Tachihara, K., Mizuno, A., \& Fukui, Y. 1999, preprint

Takayasu, H. and Suzuki, J. 1991, J. Phys. A., 24, L1309

Veneziano, D., Moglen, G. E., and Bras, R. L. 1995, Phys. Rev. E, 52, 1387

Verter, F. and Rickard, L. J. 1998, AJ, 115, 745

Vogelaar, M. G. R. and Wakker, B. P. 1994, A\&A, 291, 557

Vogelaar, M. G. R., Wakker, B. P., and Schwarz, U. J. 1991, in Fragmentation of Molecular Clouds and Star Formation, ed. E. Falgarone, F. Boulanger, and G. Duvert (Dordrecht: Reidel), p. 508 
Wakker, B. P. 1990, Ph.D. dissertation, Univ. of Groningen

Walter, F. M. and Boyd, W. 1991, ApJ, 370, 318

Westpfahl, D. J., Coleman, P. H., Alexander, J., \& Tongue, T. 1999, ApJ, 117, 868.

Whittet, D.C.B., Kirrane, T. M., Kilkenny, D., Oates, A. P., Watson, F. G., and King, D. J. 19487, MNRAS, 224, 497

Wilking, B. A., Schwartz, R. D., and Blackwell, J. H. 1987, AJ, 94, 106

Wilson, T. L. and Walmsley, C. M. 1989, A\&A Rev, 1, 141

Xie, T., Goldsmith, P. F., and Zhou, W. 1991, ApJ, 371, L81

Yamaguti, M. and Prado, C. P. C. 1995, Phys. Lett. A, 206, 318

Yonekura, Y., Mizuno, N., Saito, H., Mizuno, A., Ogawa, H., \& Fukui, Y. 2000, PASJapan, in press

Zinnecker, H., McCaughrean, M. J., and Wilking, B. A. 1993, in Protostars and Planets III, eds. E. H. Levy, J. I. Lunine (U. of Arizona Press: Tucson), p. 429 
Table 1. Properties of the Regions Studied

\begin{tabular}{lrrrrr}
\hline \hline Subregion & R. A. & Dec. & \# Res. Elem. & Dist. $(\mathrm{pc})$ & \# YSO/pc \\
\hline Tau & $4^{h} 30^{\prime}$ & $+26^{\circ} .0$ & $3.2 \times 10^{4}$ & $140^{a}$ & $0.3^{b}$ \\
R CrA W & $19^{h} 04^{\prime}$ & $-37^{\circ} .0$ & $1.0 \times 10^{4}$ & $130^{c}$ & $3.0^{d}$ \\
R CrA E & $19^{h} 32^{\prime}$ & $-37^{\circ} .0$ & $2.6 \times 10^{4}$ & $130^{c}$ & $0.0^{d}$ \\
Cham 1 & $11^{h} 00^{\prime}$ & $-77^{\circ} .0$ & $1.7 \times 10^{4}$ & $140^{e}$ & $3.5^{f}$ \\
Cham 2 & $12^{h} 40^{\prime}$ & $-78^{\circ} .0$ & $1.1 \times 10^{4}$ & $140^{e}$ & $2.0^{f}$ \\
Cham 3 & $12^{h} 20^{\prime}$ & $-80^{\circ} .0$ & $1.4 \times 10^{4}$ & $140^{e}$ & $0.0^{f}$ \\
Musca & $12^{h} 20^{\prime}$ & $-71^{\circ} .0$ & $1.5 \times 10^{4}$ & $140^{e}$ & $0.1^{f}$ \\
$\rho$ Oph & $16^{h} 26^{\prime}$ & $-26^{\circ} .0$ & $5.6 \times 10^{4}$ & $125^{g}$ & $23.0^{h}$ \\
Oph E & $16^{h} 50^{\prime}$ & $-24^{\circ} .0$ & $2.0 \times 10^{4}$ & $125^{g}$ & $\ldots$ \\
Oph W & $15^{h} 40^{\prime}$ & $-27^{\circ} .0$ & $3.6 \times 10^{4}$ & $125^{g}$ & $\ldots$ \\
Oph U & $16^{h} 26^{\prime}$ & $-19^{\circ} .0$ & $2.2 \times 10^{4}$ & $125^{g}$ & $\ldots$ \\
Oph N & $16^{h} 40^{\prime}$ & $-10^{\circ} .0$ & $1.0 \times 10^{5}$ & $125^{g}$ & $\ldots$ \\
L 134 & $15^{h} 56^{\prime}$ & $-5^{\circ} .0$ & $2.4 \times 10^{4}$ & $160^{i}$ & $0^{j}$ \\
\hline
\end{tabular}

References. - (a) Elias 1978; (b) Beckwith et al. 1990, Kenyon et al. 1990; (c) Marraco and Rydgren 1981; (d) Graham 1990; (e) Whittet et al. 1987, Franco 1991; (f) Schwartz 1989, Baud 1984; (g) deGeus et al. 1989; (h) Wilking et al. 1989; (i) Snell 1981; (j) Sargent et al. 1983. 